\title{
Aportes del Neuromarketing para la atención de personas adultas mayores en centros educativos
}

\author{
Nancy Vásquez-Flores \\ Comunicadora social, Oficina Institucional de Mercadeo y Comunicación, \\ Universidad Estatal a Distancia, Costa Rica \\ nvasquez@uned.ac.cr
}

Recibido: 27 de mayo del 2019 Corregido: 12 de agosto del 2019 Aceptado: 27 de setiembre del 2019

\section{Resumen}

En este ensayo, se realiza una discusión acerca de algunos aportes que puede brindar el neuromarkting para la atención de personas adultas mayores, en centros de enseñanza donde se brinda una oferta educativa dirigida a esta población. Como parte de los argumentos que sustentan este texto, se afirma que el neuromarkting, por estar enfocado en los procesos neurológicos y cognitivos, serviría como una herramienta para atraer y retener a adultos mayores.

Palabras clave: Neuromarketing; funciones cognitivas; persona adulta mayor; centros de enseñanza; oferta educativa.

\begin{abstract}
Contributions of Neuromarketing for the attention of older people in educational center

In this essay there is a discussion about some contributions that neuromarkting can provide for the care of older adults, in schools where an educational offer is offered to this population. As part of the arguments that support this text, it is stated that neuromarkting, being focused on neurological and cognitive processes, would serve as a tool to attract and retain older adults.
\end{abstract}

Key words: Neuromarketing; cognitive functions; older adult; teaching centers; Educative offer.

\section{INTRODUCCIÓN}

En el país crecen las demandas y necesidades relacionadas con las personas adultas mayores, pues esta población va en aumento y, por ello, se debe hacer conciencia acerca de la responsabilidad social hacia este sector.

Según datos del Instituto Nacional de Estadística y Censos (2019), en Costa Rica, hacia el año 2018, se contabilizaban 794.006 personas adultas mayores, número que ha ido en constante aumento, según se puede observar en la Tabla 1. 
TABLA 1

Incremento de la población adulta mayor en el periodo 2012-2017 en Costa Rica

\begin{tabular}{ccccc} 
& Total & \multicolumn{3}{c}{ Años } \\
2012 & 582.197 & $60-65$ & $65-74$ & 75 y más \\
2013 & 623.374 & 178.399 & 225.838 & 177.960 \\
2014 & 636.802 & 184.189 & 243.664 & 195.521 \\
2016 & 681.201 & 195.394 & 248.417 & 192.991 \\
2017 & 752.846 & 206.219 & 274.084 & 200.898 \\
\hline
\end{tabular}

Fuente: Elaboración propia a partir de datos del INEC.

*Nota: no se encontró en los registros la información sobre el año 2015.

Además, el Instituto de Estudios Sociales en Población indica que, de continuar con esa tendencia, las proyecciones para el año 2025 señalan que la población adulta mayor llegará a 864.387, lo que representará el $14.5 \%$ de quienes habiten en el país. Se trata de un fenómeno complejo, pues "el envejecimiento poblacional del país se debe a varios factores entre los que destaca el aumento en la esperanza de vida al nacer, la disminución del número de nacimientos y la disminución de la mortalidad" (Programa de Gerontología, 2014, p.3).

Según Huenchuan y Rodríguez (2010), un organismo internacional como la Comisión Económica para América Latina y el Caribe (CEPAL) establece que la vejez se considera a partir de los 60 o 65 años. Por ley, se hace referencia a las personas a partir de esta edad como "adultos mayores" o "personas adultas mayores", quienes, de acuerdo con lo que indica esa Comisión, "padecen de discriminación por su edad, provocando falta en igualdad de condiciones en derechos humanos y libertades políticas, económicas, sociales, culturales, civiles, entre otros" (p.15).

Una respuesta a esa posible desigualdad consiste en cumplir los derechos de las personas adultas mayores para garantizar su bienestar y calidad de vida. Por ejemplo, la educación debe ser parte de lo que se ofrezca a este sector de la población, asegurando su permanencia en sistemas de enseñanza dirigidos a potenciar sus capacidades para contribuir con el mejoramiento de condiciones en su vida personal y en su participación social.

Las instituciones, en forma especial, deben idear beneficios para este segmento de la población que se orienten a fomentar una mejor calidad de vida. Por ello, en Costa Rica, la Asamblea Legislativa de la República decretó, en 1999, la ley 7935, denominada Ley Integral para la Persona Adulta Mayor, la cual busca garantizar igualdad de condiciones e "impulsar la atención integral de las personas adultas mayores por parte de las entidades públicas y privadas velando por el funcionamiento adecuado de los programas y servicios destinados a esta población, entre otros" (Asamblea Legislativa de la República Costa Rica, 1999. p.7).

Por otra parte, el Consejo Nacional de la Persona Adulta Mayor (CONAPAM, 2013), ha denunciado que:

La sociedad tiende a valorar a las personas en función de cuánto aportan laboralmente a la economía del país, a eso se le conoce como productividad. En el caso de las personas adultas mayores se les considera improductivas por no estar incorporadas al proceso laboral o por estar jubiladas.

Esta forma errada de pensar en algunas sociedades occidentales como la nuestra discrimina a las personas mayores, provocándoles aislamiento e inactividad, afectándoles de manera importante en términos de salud integral y mejoramiento de su calidad de vida. 
Es necesario valorar el aporte que realizan muchas personas mayores a la producción del país por medio de su experiencia, de su consejo a generaciones más jóvenes e incluso participando en labores domésticas o de cuido a nietos o personas menores de edad (p.10).

Si bien valorar a la persona por su edad productiva puede ser discriminatorio en relación con adultos mayores, estereotipos de ese tipo influyen sobre las actitudes y, como consecuencia, afectan los ámbitos personales, familiares y nacionales causando exclusión.

Las personas excluidas, según Castro (2017), tienen "poco acceso a órganos de poder y toma de decisiones y se ven afectadas en su vida cotidiana. Las dimensiones de la exclusión social se pueden clasificar en: educativa, laboral, económica, política, de género, diversidad cultural, entre otros" (p. 11). Por el contrario, la inclusividad envuelve acciones dirigidas a garantizar la equidad, la solidaridad, la igualdad de oportunidades, la no discriminación, la consideración, entre otras.

Si bien todas las personas deben gozar de la misma igualdad y derechos, los adultos mayores pueden estar expuestos a mayores desventajas, a pesar de que hay países con normas que promueven la participación plena de este sector de la población. Como se mencionó antes, la sociedad los segrega en razón de su edad. Incluso, la distribución de los recursos para el beneficio de su desarrollo no es igual que la que se dirige a otros sectores de la población (Huenchuan \& Rodríguez, 2010).

La población adulta mayor se convierte en un grupo que puede favorecer la aparición de un gran número de profesiones y de servicios, pero eso exige la adaptación de entornos educativos, políticas y, sobre todo, de los programas o proyectos formulados en términos de acción social, extensión e investigación.

Cabe destacar que la Organización de Estados Americanos (OEA) creó, en el 2015, la Convención Interamericana sobre la Protección de los Derechos Humanos de la Personas Mayores, la cual fue aprobada, en junio del 2016, por Costa Rica, ratificando así la protección de los derechos humanos de las personas adultas mayores. Costa Rica fue unos de los primeros países en firmar el documento y le siguieron Argentina, Brasil, Chile, Uruguay y República Dominicana (Presidencia de la República, 2016).

Según lo expuesto anteriormente, este ensayo tiene por objetivo discutir cómo el neuromarketing puede ser una herramienta que, enfocada en procesos cognitivos (la percepción, la atención, la memoria, el pensamiento y el lenguaje), coadyuvaría en el ofrecimiento de una oferta educativa de calidad que satisfaga las necesidades de las personas adultas mayores, fortaleciendo con ello los procesos de inserción educativa en esta población.

\section{DESARROLLO}

\section{Panorama de la oferta educativa a nivel nacional e internacional para personas adultas mayores}

En diferentes países, se han impulsado condiciones académicas y formativas dirigidas a las personas adultas mayores, por medio de centros de educación superior públicos y privados. Estos ofrecen programas diseñados específicamente para personas que no tuvieron la oportunidad de estudiar o que desean ampliar sus conocimientos en áreas diferentes a su formación, con el fin de satisfacer sus necesidades de aprendizaje.

Francia fue uno de los precursores de las universidades de la tercera edad. Su primera iniciativa fue en 1973. Según Lemieux (1997), estas universidades se crearon para ayudar a las personas adultas mayores poniendo a su servicio recursos como aulas, profesorado u ofertas de formación, entre otros. En 1975, 
se creó en Francia la Asociación Internacional de Universidades de la Tercera Edad (AIUTA), la cual incorpora, actualmente, más de 3000 programas educativos en diferentes universidades del mundo. También, existen la Federación Internacional de Asociaciones de Personas de Edad (FIAPA) y la Eurolink Age (Red Europea de Personas Adultas Mayores, Cultura y Arte). A partir de esa experiencia francesa, se formaron más programas en el resto de los países europeos y Estados Unidos.

En Estados Unidos, uno de los más novedosos ha sido el llamado Elderhostel, creado en 1975. Es un programa que ofrece la estancia en centros educativos de ese país y en el extranjero. Para 1980, ya contaba con más de 20,000 adultos mayores que participaron en procesos de aprendizaje en cincuenta estados del país y la mayor parte de Canadá y, hacia 1981, se expandió la experiencia hacia Gran Bretaña y Escandinavia.

En 2010 y desde sus inicios, el programa Elderhostel había atendido a más de 4 millones de adultos mayores y la organización cambió de marca con un nuevo nombre: Road Scholar. Hoy atiende a más de 100.000 estudiantes, que combinan los viajes con la educación (Roadscholar, 2017).

Las aulas de la tercera edad son centros de enseñanza donde la persona adulta mayor organiza su tiempo libre, por medio de actividades formativas para desarrollar y engrandecer sus relaciones interpersonales. El caso más emblemático es el de España, con el programa denominado "Guías voluntarios de la tercera edad", para enseñar en los museos a niños, jóvenes y jubilados una vez que ellos hayan sido capacitados (Guirao \& Sánchez, 1997).

En Costa Rica, las universidades estatales deben asumir un papel significativo en el tema de la inclusión de personas adultas mayores en procesos educativos. Para ello, según Guirao \& Sánchez (1997), es necesario tomar en cuenta las siguientes razones:

- Razones sociales: es obligación de las universidades ofrecer una respuesta solidaria y servicial al aumento de un grupo de personas que se encuentren liberadas de sus obligaciones profesionales o laborales.

- Razones institucionales: es el rol que debe tener la universidad con la población como proveedora de conocimiento cuando la necesidad de formación se hace más evidente.

Todo esto supone un reto para el sistema educativo costarricense $y$, para ello, las cinco universidades estatales pueden suplir las demandas del sector, en mayor o menor medida, según diferentes tipos de oferta:

- Servicios educativos: se desarrollan actividades culturales de tiempo libre con el fin de entretener y contribuir con las relaciones sociales entre las personas adultas mayores.

- Actividades educativas: contribuye a que los adultos mayores logren resolver sus problemas sociales existentes, gracias al conocimiento y aporte de la enseñanza.

- Programas educativos: son estudios formales que contienen un plan de estudios con todas las características de la enseñanza superior (Lemieux, 1996).

Puntualmente, las universidades públicas costarricenses cuentan ya con diferentes programas dirigidos a la población adulta mayor: el Programa Institucional para la Persona Adulta y Adulta Mayor (PIAM), de la Universidad de Costa Rica; el Proyecto Educativo para la Persona Adulta Mayor (PAMTEC), del Tecnológico de Costa Rica; el Programa de Atención Integral de la Persona Adulta Mayor (PAIPAM), de la Universidad Nacional; el Programa de Gerontología, de la Universidad Estatal a Distancia y el Programa Ágape, de la Universidad Técnica Nacional.

Esta amplia oferta educativa en el país hace que las universidades públicas, como parte de su responsabilidad social y permanente mejora de la oferta educativa ofrecida a las personas adultas mayores, deba 
supervisar la calidad de los servicios que se ofrecen a este sector poblacional. Según Stanton (2013), "la meta a futuro para las instituciones de servicios es aumentar la productividad y medir cómo se satisfacen las necesidades" (p.584). En este sentido, una parte relevante para el mejoramiento continuo en la atención a las necesidades de las personas adultas mayores debe enfocarse en las características propias de sus procesos cognitivos. Y para ello, las neurociencias, en especial el neuromarketing, pueden ser herramientas fundamentales para comprender muchos de los aspectos neurológicos relacionados con el aprendizaje en esta etapa de la vida.

\section{Aspectos neurológicos del aprendizaje en la persona adulta mayor}

El sistema nervioso está formado por neuronas y células gliales y cada una cumple una función. Así, la neurona recibe y transmite información, mientras que las gliales sostienen el tejido nervioso y realizan otras actividades nutricionales y de facilitación de la comunicación neuronal. Es importante resaltar que el cerebro tiene alrededor de 86 mil millones de neuronas, las cuales se comunican unas con otras en el proceso denominado sinapsis. El cerebro también es el responsable de los procesos mentales y cognitivos, entre los cuales se puede mencionar la percepción, la atención, la memoria, el pensamiento y el lenguaje.

La sinapsis permite que el ser humano tenga estímulos (como el dolor o el placer), dando lugar así a la mediación de procesos afectivos. Es por ello que esta característica neurológica permitiría darle mayor valor a una acción de compra, según los estímulos sinápticos. Por ejemplo, cuando un adulto mayor conoce sobre cierto servicio, en este caso la oferta educativa formal o cursos libres de un centro de enseñanza, sus circuitos neuronales trasladan la información en una señal eléctrica para que esa información llegue al cerebro y active el interés por la oferta de aprendizaje.

Por su parte, el aprendizaje es la capacidad de cambiar la conducta a partir de las experiencias, por lo cual ser humano podrá adaptarse a nuevas situaciones ambientales y sociales. Esto se da por la memoria, que es un fenómeno que se obtiene a partir de un sentido de continuación (Espinoza, Oruro, Carrión \& Aguilar, 2015). Por ejemplo, si un adulto mayor se jubila, y dado que ha venido trabajando por, al menos treinta años, aproximadamente, su cerebro actúa por medio de plasticidad (modo donde el sistema nervioso cambia a partir de su interacción con el entorno) y memoria, pues está acostumbrado a vivir bajo una rutina o ritmo de trabajo a lo largo de los años. Sin embargo, si esta persona, en su retiro, no cuenta con un proyecto de vida, su sistema nervioso se verá afectado, debido a que, aunque el cerebro está activo hasta el día en que un ser humano fallece, puede sufrir degeneración y causar limitaciones a nivel emocional y físico. Así, León (2018) advierte que cuando una persona no hace conexiones sinápticas, es como si estuviera muriendo y, por ello, recomienda a las personas involucrarse en actividades para tener una mente lúcida.

En referencia al deterioro intelectual, el Consejo Nacional de la Persona Adulta Mayor (CONAPAM, 2013) menciona que:

Las personas adultas mayores tienen tanta capacidad para aprender como cualquier otra persona. De igual forma, las enfermedades mentales no son propias de la vejez y su incidencia en esta etapa de la vida no puede generalizarse. A pesar de que existe una actitud social negativa en relación al aprendizaje en la vejez, los estudios confirman que la adquisición y asimilación de nuevos conocimientos, comportamientos, aptitudes, actitudes o hábitos se pueden dar en cualquier edad. Lo que puede modificarse en la vejez es el tiempo o la velocidad de la asimilación. Es decir, lo que cambia es la agilidad, pero no la habilidad. Toda persona, a cualquier edad, puede verse afectada en su 
capacidad de aprendizaje. Es un error pensar que todas ellas son olvidadizas o que, tarde o temprano, tendrán una incapacidad mental (p. 7).

De igual forma, los neurocientíficos afirman que el cerebro puede mantenerse saludable y con un buen funcionamiento a medida que se envejece. Las causas del deterioro de la memoria o la inteligencia entre otras facultades mentales son las enfermedades (Society for Neurosciense, 2008). También, indican que "las tecnologías han contribuido a la exploración y función del cerebro con más detalle que años atrás es así como los neurocientíficos son cada vez más capaces de distinguir los procesos del envejecimiento normal y de la enfermedad" (p. 34)

La corteza cerebral es donde ocurren las sinapsis y está dividida en dos hemisferios que, a su vez, se dividen en lóbulos que tienen funciones específicas:

- Lóbulo frontal: donde se toman las decisiones. Nos permite fijar la atención, memorizar datos y regula las emociones y motivación.

- Lóbulo parietal: mezcla los datos de lo que se ve, escucha o se habla para que parezca una experiencia perceptiva completa, así como el razonamiento espacial, el movimiento del cuerpo y la orientación.

- Lóbulo occipital: tiene que ver con la percepción y reconocimiento de lo que se ve.

- Lóbulo temporal: regula las emociones, estados de ánimo, etc., articula el lenguaje y la comprensión de los sonidos y las voces.

- I Ínsula: se vincula con el control de vísceras y órganos (Sabater, 2018).

De tal manera, se puede indicar que la atención, las emociones y memoria, así como la inteligencia y afectividad o empatía, son procesos cognitivos, y a ellos se les puede denominar como parte de un modelo persuasivo de marketing que puede influir por medio de un aprendizaje consciente y voluntario en temas de publicidad dirigida al adulto mayor, para generar motivación hacia ciertos productos o servicios.

A continuación, se abordará de manera más explicativa acerca del modelo persuasivo de marketing, el cual se denominará neruomarketing.

\section{El Neuromarketing}

Es una nueva área de la investigación multidisciplinaria donde se aplican los conocimientos de la neurociencia en procesos cognitivos y afectivos a estímulos de marketing (Linares, 2018). Por lo tanto, "puede verse como una extensión de la búsqueda de la cuantificación y la certeza en aspectos previamente indefinidos del comportamiento humano" (Fischer, Chin \& Klitzman, 2011 p.1).

También, se puede definir según Perdigones (2017) como:

El estudio del cerebro de manera multidisciplinar que otorga un apoyo a la psicología con el objetivo de entender la complejidad del funcionamiento mental, ayudando a respaldar y contrastar, a través de las herramientas de marketing y del comportamiento del consumidor, las diferentes aplicaciones del marketing y así delinear las estrategias que debe seguir la organización" (parrs. 2-3).

Por otra parte, Braidot (2005) señala que es el estudio del funcionamiento del cerebro y de la neurona en el sistema nervioso, brindando conocimiento y herramientas esenciales para entender las enfermedades 
del sistema nervioso. Para Álvarez (2011), el término neuromarketing describe la aplicación de métodos neurocientíficos, por medio del uso del laboratorio y se aplica al mundo del marketing para analizar y comprender el comportamiento humano y sus emociones en relación con el mercado. En resumen, se puede indicar que es la disciplina que estudia el funcionamiento del cerebro, con el fin de crear estrategias efectivas de marketing para la toma de decisiones por parte del consumidor.

Según se ha mencionado, el cerebro crea los pensamientos, esperanzas, sueños e imaginación del ser humano (Society for Neurosciense, 2008). Es así que se parte que los conocimientos básicos del neuromarketing que se enfocan en el aprendizaje y la memoria, gracias a los cuales la información se codifica, se almacena y queda disponible para su reutilización en el momento preciso para la toma de decisiones del consumidor.

En neuromarketing, existen ciertas herramientas para medir o conocer las emociones o el estado motivacional. En este caso puntual, se podría hacer uso de estos con los adultos mayores como un protocolo de investigación. Con los resultados que estas herramientas arrojen, se podrá identificar cuáles son los cursos de su mayor agrado. Entre esas herramientas están: eye tracking, visor de realidad virtual, tobi glasses, biometrias, electroencefalografía, EEG gadgets.

La persona adulta mayor dará su lealtad de compra a aquellas instituciones que le demuestren un genuino interés y un trato diferencial, pues esto crea una emoción o sentimiento positivo. Por lo tanto, es importante la atención que se le proporcione, pues de eso dependerá el poderle mantener o retener en una institución educativa y afianzar su participación en los cursos que se ofrezcan.

Desde el punto de vista del neuromarketing, se da mayor énfasis en proporcionar emociones a los propósitos del marketing y desde una perspectiva como las neurociencias, sin que esto signifique sea el único factor que ejerza su influencia en la decisión definitiva del consumidor. También, se debe considerar si el marketing mix (mezcla de mercadeo) es el adecuado en cuanto a precio, tipos de cursos, si se ofertan por todo el país, entre otros, con el fin de que sea de mayor interés para el adulto mayor.

Como parte del modelo persuasivo de neuromarketing, esto podrá generar un impacto para que el adulto mayor se sienta motivado en matricular cursos, tomando en cuenta sus estados afectivos. Algunas recomendaciones para esto son:

- Escuchar a las personas adultas mayores con mucha atención y demostrarles interés por la situación que presentan.

- Mostrar cordialidad para solventar la situación, así como la manera de responderles. Siempre hay que expresarles comprensión. La cortesía es muy importante.

- Cuidar las expresiones. Estas se dan en el sistema nervioso autónomo, donde se contienen todas las neuronas que transportan mensajes entre el sistema nervioso central y los órganos internos. Además, interviene en la experiencia de varias emociones (Aguilar, 2018), que reciben con la atención ya sea verbal, corporal y afectiva.

Justamente, en Costa Rica, en el año de 1988, por medio de un Decreto Ejecutivo del Ministerio de Trabajo (26991-MTSS- 22, mayo 1998) se ordenó a todas las instituciones públicas brindar atención preferencial a la población adulta mayor y que se dispusiera de las facilidades necesarias. Sin embargo, aún se comenten una serie de errores con la atención, como por ejemplo:

1. No tener personal entrenado para brindar una buena atención al adulto mayor.

2. Tratar de ganar una discusión. Siempre se debe procurar resolver la situación y no ponerse a discutir con el adulto mayor.

3. Si le ofrece algo al adulto mayor, debe cumplirlo y no hacerle falsas esperanzas. 
4. No contar con un registro actualizado de datos o información del adulto mayor.

5. Los adultos mayores en ocasiones deben incurrir en pasos innecesarios para llegar a ser atendido. Es muy usual que lo dirijan a una oficina, luego a otra y otra, y en ninguna se soluciona la situación.

6. No escuchar o estar interrumpiendo al adulto mayor sin permitir que ellos se comuniquen o desahoguen con lo que necesitan.

En ocasiones se trata con prejuicios o ideas y caracterizaciones negativas a las personas adultas mayores (Bousfield \& Hutchisdon, 2010). Y para evitar eso, la comunicación es esencial en el trato que se le dé a esta población, pues esto contribuirá a influir positivamente si se tienen ciertas atenciones y un trato respetuoso que refuerce los estímulos cognitivos a partir del aprendizaje, la memoria, las emociones y, por ende, el interés por el producto o servicio que se ofrece.

También se recomienda hacer uso de palabras que dignifiquen a la persona adulta mayor, evitando comunicaciones que puedan ser despectivas y estigmatizantes en relación con esta población, por ejemplo:

- Ciudadano de oro

- Viejo

- Abuelito

- Anciano

Por el contrario, conviene referirse usando el nombre de la persona o un trato formal como señor o señora. Esto es relevante, no solo como una forma de respeto hacia las personas adultas mayores, sino también como muestras de cordialidad y lealtad que calarían en sus emociones, como parte de una estrategia de neuromarketing para posicionar un producto o servicio. Además, es importante que siempre se brinde atención con una sonrisa, lo cual hace más agradable la primera impresión, no solo de la persona que les atenderá, sino de la institución como tal.

\section{Aportes y desafíos del neuromarketing}

El neuromarketing ha venido a ser un elemento revolucionario para los negocios, dado que utiliza técnicas de mercadeo para sustentar los comportamientos de los diferentes tipos de consumidores, según sus preferencias y necesidades, describiendo esa conducta de la persona a la cual se dirige la estrategia de venta generando un impacto emotivo en él y favoreciendo al marketing en cuanto a las estrategias de comunicación, precio, producto, distribución, promoción, entre otros, generándole grandes beneficios de inversión y utilidad a las empresas, tal como se ha mencionado anteriormente.

Por otra parte, también ha sido un tema sensible en su aplicación, debido a que ha generado una serie de controversias y fundamentos éticos entre los investigadores (Dinu, 2013). Así, según Farah, Smith, Gawuga, Lindsell y Foster (2010), las reacciones pueden asociarse con la metodología de investigación, pues podría tener algún tipo de influencia en los procesos cerebrales de las personas, esto con el uso de las herramientas de neuromarketing, donde podrían provocar que se manipule la información o los resultados obtenidos, comprometiendo la capacidad del individuo para tomar decisiones de compra.

Por eso, a continuación, se exponen algunos cuidados y consideraciones éticas a tomar en cuenta en relación con la población adulta mayor y la promoción de procesos educativos:

- Los investigadores o especialistas en mercadeo tienen prohibido engañar a los participantes en el estudio, ya sea por desconocimiento o la falta de información en el campo de la neurociencia. 
- A las empresas no se les permite atraer a los consumidores con promociones de mercadeo para usar después en otros estudios.

- Los objetivos del estudio deben ser claramente comunicados y los datos grabados no deben mantenerse más de lo que se necesita para terminar la investigación.

- Los participantes tienen el derecho de interrumpir el estudio y exigir que su información sea borrada si se sienten afectados por su contenido (Neuromarketing Sciense and Business Association, s.f.).

Para que esta normativa se cumpla, se estableció, en el 2012, la Asociación de Neuromarketing Sciense and Business Association (NMSBA), para promover el campo del neuromarketing. En menos de una década, se desarrolló como una comunidad internacional con miembros en más de cien países. La misma elaboró un Código de Ética, que todos sus miembros están obligados a cumplir. Parte de sus principales responsabilidades es la contribución para el desarrollo y la puesta en práctica de normas internacionales y la estandarización de la disciplina.

Como inicialmente se ha venido abordando, el adulto mayor es un segmento de la población vulnerable ante ciertos estímulos y, por ello, el tema de la ética en el neuromarketing es importante para que este sector de la población no se vea influenciado o manipulado ante estudios de neuromarketing.

\section{REFLEXIONES FINALES}

Se ha evidenciado que es una obligación de las instituciones y universidades ofrecer respuestas solidarias hacía la población adulta mayor ante la situación de que esta población va en aumento. Los centros de enseñanza deberán comprometerse en ser más inclusivos hacia la persona adulta mayor para que ellos encuentren oportunidades de desarrollo y, con ello, mejores condiciones de vida, en donde a su vez reciban un buen trato de parte de quienes les rodean.

Justamente se evidenció que las universidades tienen la normativa y lineamientos para favorecer a la población adulta mayor; sin embargo, sus programas aún no aplican experiencias de universidades del extranjero las cuales han tenido mucho éxito.

El país debe dar respuesta en cuanto a abogar y ser ejemplo en el tema de los derechos humanos para la persona adulta mayor ante la OEA y las universidades deberán dar el ejemplo como centros de enseñanza.

La atención deberá ser una de las razones prioritarias para cualquier institución; debe ser una meta a cumplir y parte de los valores de la cultura organizacional. Esto será un factor clave de aprendizaje.

Según el tema de discusión acerca de cómo los centros de enseñanza podrían coadyuvar a la persona adulta mayor en ofrecer una oferta educativa con una atención de calidad, haciendo uso de las estrategias de neuromarkting, se ha concluido que esta es una disciplina que ofrece un gran potencial en el mundo de la investigación del comportamiento del consumidor y del marketing, al hacer uso de la neurociencia a través de la colaboración entre científicos para comprender con mayor precisión el comportamiento humano en general, haciendo que el funcionamiento del cerebro respalde las herramientas tecnológicas de marketing con base a diagnósticos para medir los comportamientos de los consumidores.

Gracias al neuromarketing, se podría evaluar al adulto mayor en cuanto a lo que piensa y siente de manera más certera. Sobre todo, y con base a lo expuesto en este ensayo donde se indica que los estímulos que ocurren en el cerebro hacen que se activen deseos de compra, además, proporciona datos más exactos para la realización de campañas publicitarias y estrategias de venta donde se podrá influir en las emociones, estados de ánimo y memoria como parte de la decisión de compra al adquirir un bien o servicio. 
El neuromarketing contribuye a medir estímulos, sin la necesidad de preguntarle a la persona o consumidor, por medio de distintas herramientas, entre ellas: eye tracking, visor de realidad virtual, tobi glasses, biometrias, electroencefalografía, EEG gadgets, entre otros Sobre todo, aplica los principios y conocimientos de la neurociencia, lo que hace que se genere entendimiento del pensamiento del consumidor, lo cual podría convertirla en una herramienta clave para realizar investigación de mercados.

Por otra parte, el tener acceso a la información por medio de las herramientas del neuromarketing, produce un lanzamiento exitoso de productos o servicios por medio del uso de una publicidad más asertiva según lo que espera el consumidor. Y gracias a estos estudios, las empresas pueden mejorar los procesos, reducen costos y existe menor riesgo para el desarrollo o implementación de sus estrategias de marketing.

El neuromarketing puede avanzar cada vez más en la medición de la parte emocional del adulto mayor en el proceso de compra. Otro de los beneficios o recomendaciones de esta disciplina es enfocar los mensajes de las campañas en las emociones con el fin de generar sensibilidad y empatía, de tal forma los resultados serán mejores. En este caso puntual, mejorarían los procesos de matrícula de oferta educativa para el adulto mayor incrementando la misma.

Asimismo, los centros educativos deberían considerar el establecimiento de protocolos para la atención del adulto mayor por medio de capacitaciones a funcionarios en cuanto a las leyes que rigen la atención y servicios y así cumplir con la normativa que se exige, no solo como una responsabilidad país, sino como parte de las estrategias de neuromarketing para estimular los procesos de compra.

Ahora, es importante siempre tener ética empresarial ante los estudios o investigaciones que se desarrollen por medio de las herramientas de neuromarketing. De manera concreta, la estimulación cognitiva en el adulto mayor se desarrolla por medio de la plasticidad del cerebro que este se adapte a las situaciones que se puedan presentar, modificando las necesidades a través de un mejor uso de las redes neuronales. Esto significa que, si una empresa que hace estudios de neuromarketing altera o manipula los resultados, podría provocar en el adulto mayor que varíen sus habilidades cognitivas (atención, concentración, el razonamiento, las emociones, entre otros), produciendo que la persona cambie su preferencia o decisión de compra.

El desarrollo del neuromarketing debe estar controlado para evitar malas prácticas y abusos. Además, se debe respetar la integridad de la persona, su privacidad y sensibilidad.

\section{REFERENCIAS}

Álvarez, R. (2011). Fusión perfects. Neuromarketing. Seducir al cerebro con inteligencia para ganar en tiempos exigentes. Madrid: Pearson.

Asamblea Legislativa de la República Costa Rica. (1999). Ley integral para la persona adulta mayor. Recuperado de http://www.imprentanacional.go.cr/editorialdigital/libros/textos\%20juridicos/ ley_persona_adulta_mayor_edincr.pdf

Braidot, N. (2000). Neuromarketing: ¿Por qué tus clientes se acuestan con otros si dicen que les gustas tú? Barcelona: Gestión 2000.

Bousfield, C. \& Hutchison, P. (2010). Contact, anxiety and young people's attitudes and behavior intentios towars the elderly. Educational Gerentology, 36, 451-466. Recuperado de https://www.tandfonline.com/doi/full/10.1080/03601270903324362?scroll=top\&needAccess=true

Bustos, A., Padilla, R., Pernudi, V.,Sandoval, I. \& Solorzano N. (2005). Percepciones de la población costarricense acerca de la población adulta mayor, Pulso Nacional, Universidad Nacional, Instituto 
Estudios Sociales en Población. 1-16. ISSN 1659-0007. Recuperado de: https://www.conapam.go.cr/ mantenimiento/percepcionesdelapoblacioncostarricense2005.pdf

Castro, M. (2017). La educación a distancia como factor de inclusión social: UNED 40 años democratizando la educación en Costa Rica. Espiga. 16, 8-39. doi: 10.22458/rev16i0.1926. Recuperado de: http:// investiga.uned.ac.cr/revistas/index.php/espiga/article/view/1926/2167

Consejo Nacional de la Persona Adulta Mayor. (2013) Mitos y estereotipos acerca del envejecimiento y la vejez. San José, C.R.: CONAPAM. Recuperado de http://www.conapam.go.cr/mantenimiento/ FOLLETO\%20MITOS.pdf

Dinu, V. (2013). Protection of consumer rights in the field of economic services of general economic interest. Amfiteatru Economic. XV(34), 309-310. Recuperado de: https://papers.ssrn.com/sol3/papers. cfm?abstract_id=2304774

Espinoza, G., Oruro, E., Carrión, D. \& Aguilar, L. (2015). Aprendizaje, memoria y neuroplasticidad. I Congreso Mundial de Neuroeducación ASEDH - CEREBRUM. Laboratorio de Neurociencia y Comportamiento. Universidad. Peruana Cayetano Heredia, Lima, Perú.

Farah, M., Smith, M., Gawuga, C., Lindsell, D. \& Foster, D. (2010). Brain Imaging and Brain Privacy: A Realistic Concern? Scholarly Commons, University ofPennsylvania.309-310. Recuperado de https://repository. upenn.edu/cgi/viewcontent.cgi?referer=https://www.google.com/HYPERLINK "https://repository. upenn.edu/cgi/viewcontent.cgi?referer=https://www.google.com/\&httpsredir=1\&article=1066\&context=neuroethics_pubs"\&HYPERLINK "https://repository.upenn.edu/cgi/viewcontent. cgi?referer=https://www.google.com/\&httpsredir=1\&article=1066\&context=neuroethics_pubs"httpsredir=1HYPERLINK "https://repository.upenn.edu/cgi/viewcontent.cgi?referer=https://www. google.com/\&httpsredir=1\&article=1066\&context=neuroethics_pubs"\&HYPERLINK “https://repository.upenn.edu/cgi/viewcontent.cgi?referer=https://www.google.com/\&httpsredir=1\&article=1066\&context=neuroethics_pubs"article=1066HYPERLINK "https://repository.upenn.edu/cgi/ viewcontent.cgi?referer=https://www.google.com/\&httpsredir=1\&article=1066\&context=neuroethics_pubs"\&HYPERLINK "https://repository.upenn.edu/cgi/viewcontent.cgi?referer=https://www. google.com/\&httpsredir=1\&article=1066\&context=neuroethics_pubs"context=neuroethics_pubs

Fischer, C., Chin, L. \& Klitzman, R. (2011). Defining Neuromarketing: Practices and Professional Challenges. PMC Us National Library of Medicine National Institutes of Health. 230-237. doi: 10.3109/10673229.2010.496623. Recuperado de https://www.ncbi.nlm.nih.gov/pubmed/20597593

Guirao, M. \& Sánchez, M. (1997). Los programas universitarios para mayores en España. En A. Lemieux. Los programas universitarios para mayores: enseñanza e investigación. Madrid: IMSERSO. Pp. 145153. Recuperado de http://www.imserso.es/InterPresent1/groups/imserso/documents/binario/ idi66_06ubarcelona.pdf

Huenchuan, S. \& Rodríguez, L. (2010). Envejecimiento y derechos humanos: situación, perspectivas de protección. Comisión económica para América Latina y el caribe (CEPAL). Santiago: Naciones Unidas. Recuperado de https://repositorio.cepal.org/bitstream/handle/11362/3803/1/lcw353_es.pdf

Instituto Nacional de Estadística y Censo. (2019). Estadísticas población adulta mayor. Recuperado de http://www.inec.go.cr/social/poblacion-adulta-mayor

Lemeux, A. (1997). Los programas universitarios para mayores: enseñanza e investigación. Madrid: IMSERSO.

León, R. (2018). Curso: Neurociencia, estrategia y marketing. Procesos de atención, aprendizaje y memoria aplicados al Marketing. Laboratorio de Neurociencia y Comportamiento. Curso llevado a cabo en la Universidad Cayetano de Heredia, Lima, Perú.

Linares, J. (2018). Curso: Neurociencia, estrategia y marketing. Neurociencia aplicada al Marketing, la publicidad y los negocios. Laboratorio de Neurociencia y Comportamiento. Curso llevado a cabo en la Universidad Cayetano de Heredia, Lima, Perú.

Neuromarketing Sciense and Business Association. (s.f.). NMSBA Code of Ethics. Recuperado de https:// www.nmsba.com/buying-neuromarketing/code-of-ethics 
Perdigones, A. (2017). Neuromarketing: concepto y aplicación. Esan Business. Recuperado de https://www. esan.edu.pe/conexion/actualidad/2017/02/06/neuromarketing-concepto-y-aplicacion/

Presidencia de la República. (Julio, 2016). Costa Rica aprueba la Convención Interamericana sobre la Protección de los Derechos Humanos de las Personas Mayores. Recuperado de http://presidencia. go.cr/comunicados/2016/07/costa-rica-aprueba-la-convencion-interamericana-sobre-la-proteccion-de-los-derechos-humanos-de-las-personas-mayores/

Programa de Gerontología. (2014). Informe de labores año 2014. Universidad Estatal a Distancia. Recuperado de: http://www.uned.ac.cr/transparencia/images/Informe_2014/pdfs/VicAcademica /23_IL_Gerontologia.pdf

Roadscholar (s.f). The Leader in Educational Travel for Adults Since 1975. Recuperado de https://www. roadscholar.org/about/our-story/

Sabater, V. (2018). Lóbulos cerebrales: características y funciones. Recuperado de: https://lamenteesmaravillosa.com/lobulos-cerebrales-caracteristicas-y-funciones/

Society for Neurosciense. (2008). Brain Fact: apuntes sobre el cerebro y el sistema nervioso. México: Universidad Veracruzeña.

Stanton W., Etzel M. \& Walker B. (1999). Fundamentos de marketing. México: Editorial ULTA. 